\title{
Is Levosimendan Infusion Useful in Patients with Advanced Heart Failure? Meta-Analysis and Systematic Review
}

\author{
Angel Alberto García Peña*, María Fernanda Buitrago Rodriguez, Ana Maria Baron and Alejandro Mariño \\ Department of Cardiology unit, Hospital Universitario San Ignacio, Pontificia Universidad Javeriana, Bogotá, Colombia
}

Received: 22 May, 2018 | Accepted: 25 Jun, 2018 | Published: 29 Jun, 2018

*Corresponding author: Angel Alberto García Peña, Department of Cardiology-Epidemiology, Hospital Universitario San Ignacio, Pontificia Universidad Javeriana, Bogotá, Colombia, Tel: 057(1)5946161, Ext: 2900; E-mail: angel.garcia@javeriana.edu.co

Citation: García Peña ÁA, Buitrago Rodriguez MF, Baron AM, Mariño A (2018) Is Levosimendan Infusion Useful in Patients with Advanced Heart Failure? Meta-Analysis and Systematic Review. J Hear Health 5(1): dx.doi.org/10.16966/2379-769X.147

Copyright: ㄷ 2018 García Peña ÁA, et al. This is an open-access article distributed under the terms of the Creative Commons Attribution License, which permits unrestricted use, distribution, and reproduction in any medium, provided the original author and source are credited.

\begin{abstract}
Background: Heart failure is a growing problem with a progressive increase in the number of patients as a result of better treatment outcomes and medical interventions. Patients with advanced heart failure who are not candidates for heart transplantation or left ventricular assist device become a major challenge for the health system, as they have a high mortality and hospitalization rate. Intermittent levosimendan infusion has been used in this group of patients even though the evidence is still controversial.
\end{abstract}

Objective: The purpose of this study is to determine the efficacy of intermittent levosimendan infusion in patients with advanced heart failure.

Methods: We conducted a systematic review of the literature on EMBASE, EPISTEMONIKOS, MEDLINE/Pubmed and Trip database until January 2018. We included published randomized clinical trials and meta-analysis without language restriction.

Results: Eleven studies including 586 patients evaluating intermittent levosimendan infusions in patients with advanced heart failure were included for evaluation. After a follow up period between 3 and 12 months there was a 55\% reduction in mortality Odds Ratio (OR) $0.45 \mathrm{Cl} 95 \%(0.26 ; 0.78$. $p<0.003)$, a reduction in natriuretic peptide levels and an improvement in left ventricular ejection fraction without a reduction in hospitalization rates.

Conclusions: Intermittent levosimendan infusions in patients with advanced heart failure showed significant benefit in patients with advanced heart failure with mortality reduction becoming a promising alternative in the treatment of this group of patients.

Keywords: Heart failure; Mortality; Inotropic agents

\section{Introduction}

The number of patients with heart failure has grown worldwide as a result of an increase in the life expectancy and the improvement in medical treatment and interventions that have shown to reduce mortality [1]. Patients with advanced heart failure who are not candidates for advanced therapy such as heart transplantation or Left Ventricular Assist Devices (LVAD) become a major challenge for the physicians because of the lack of symptomatic improvement despite neurohumoral blockage and diuretic use. Intermittent infusions of inotropic drugs are an alternative but their use is still controversial $[2,3]$.

Levosimendan is a calcium sensitizer inotrope with demonstrated improvement in left ventricular performance. The recommended infusion dose is between 0.1 and $0.2 \mu \mathrm{g} / \mathrm{Kg} / \mathrm{min}$ with or without a bolus every two to four weeks [3-5]. The cost of this therapy is elevated and therefore it is important to evaluate the benefits and select properly the patients who are candidate for levosimendan infusion [6-10].

The purpose of this study is to determine the efficacy of intermittent levosimendan infusion in patients with advanced heart failure in reducing mortality and heart failure rehospitalization and to assess the impact of intermittent levosimendan administration on left ventricular ejection fraction and natriuretic peptide levels.

\section{Methods}

\section{Protocol}

We conducted a systematic review and meta-analysis of randomized controlled trials assessing levosimendan use in patients with advanced heart failure, following the recommendation of the Cochrane Handbook for Systematic Reviews of Interventions [11].

\section{Data collection}

Two different reviewers searched studies on EMBASE, EPISTEMONIKOS, MEDLINE/Pubmed and Trip database until January 2018 without language restriction (No limits were used for the search). We also searched references from the articles chosen from the databases if they were useful. The MeSH terms used were "advanced heart failure", "heart failure", "levosimendan", "randomized controlled trial", "intermittent infusion", and "controlled trial.

\section{Inclusion and exclusion criteria}

We included Randomized Controlled Trials (RCT) published in 
full text that included adult patients older than 18 years with advanced heart failure (ischemic or non-ischemic cardiomyopathy), candidates for intermittent inotrope infusion. The trials evaluated levosimendan infusion with or without bolus for at least three months. Placebo or other medication with an inotropic effect was accepted as comparators. Non-randomized, unpublished or trials that had an incomplete follow up were excluded.

\section{Outcomes}

The primary outcome was all cause mortality. Secondary outcomes were change in the ejection fraction, change in natriuretic peptides and rehospitalization.

\section{Study selection}

Four reviewers screened the articles selecting them by title and abstract. We evaluated full text documents in a paired way excluding duplicated articles. For the analysis of the trials we followed the Preferred Reporting Items for Systematic reviews and Meta-Analyses (PRISMA) guidelines for systematic reviews and meta-analyses (Figure 1). The evaluation of the quality of the studies was performed independently by two researchers using SIGN criteria [12]. All discrepancies were identified and resolved by consensus or with a third investigator if needed. Only references of high or acceptable quality were included. The bias risk assessment was performed for each trial by two independent investigators following the Cochrane Handbook for Systematic Reviews of Interventions (Higgins 2011) criteria [13]. We evaluated the randomization domains, assignment, blinding of participants and outcome evaluation, management of missing data and selective reporting results. Each potential source of bias was rated as "low risk," "high risk" or "unclear risk." Studies were rated as high risk of bias if any of the domains was classified as high risk of bias (Figure 2).

\section{Statistical analysis}

We evaluated the baseline characteristics of the patients in the intervention and control group, the infusion protocol, the follow up duration and the outcomes of the different trials. For the treatment effect estimation we followed the Cochrane Handbook for Systematic Reviews of Interventions recommendations [13]. We extracted event rate, Hazard Ratios (HR), and 95\% Confidence Intervals (CI) for the primary and secondary outcomes. For continuous outcomes we evaluated the net difference and measurement difference with a CI of $95 \%$. For heterogeneity assessment, we performed chi2 and I2 statistic. If no heterogeneity was found, a fixed effects model was used for the meta-analysis. In case of high heterogeneity, a random effects model was proposed. Revman $5.2^{\circ}$ software was used for data processing.

\section{Results}

We identified 187 studies excluding 171 by title and abstract. The abstract and full text of the fifteen remaining studies was analyzed [1428]. We excluded one meta-analysis that included non-randomized trials [10], one study with unpublished final results publication [27], and two non-randomized trials [25,26] (Figure 1). Eleven studies were included in the analysis with a total of 586 patients, 336 in the levosimendan group and 250 in the control group [14-19,21-24,28]. Baseline characteristics are shown in table 1 . Seven studies reported mortality data (413 patients) [14-19,28]. The follow up time was between 3 and 12 months.

The clinical heterogeneity was related with the infusion protocol, follow up time and clinical scenario that guided the levosimendan infusion. Levosimendan was administered by intermittent intravenous infusions between 0.1 and $0.4 \mu \mathrm{g} / \mathrm{kg} / \mathrm{min}$ with a bolus dose between 6 and $12 \mathrm{mcg} / \mathrm{kg}$ except in four trials which did not use bolus $[14,16,18,28]$. The infusion duration time was between 6 and 24 hours

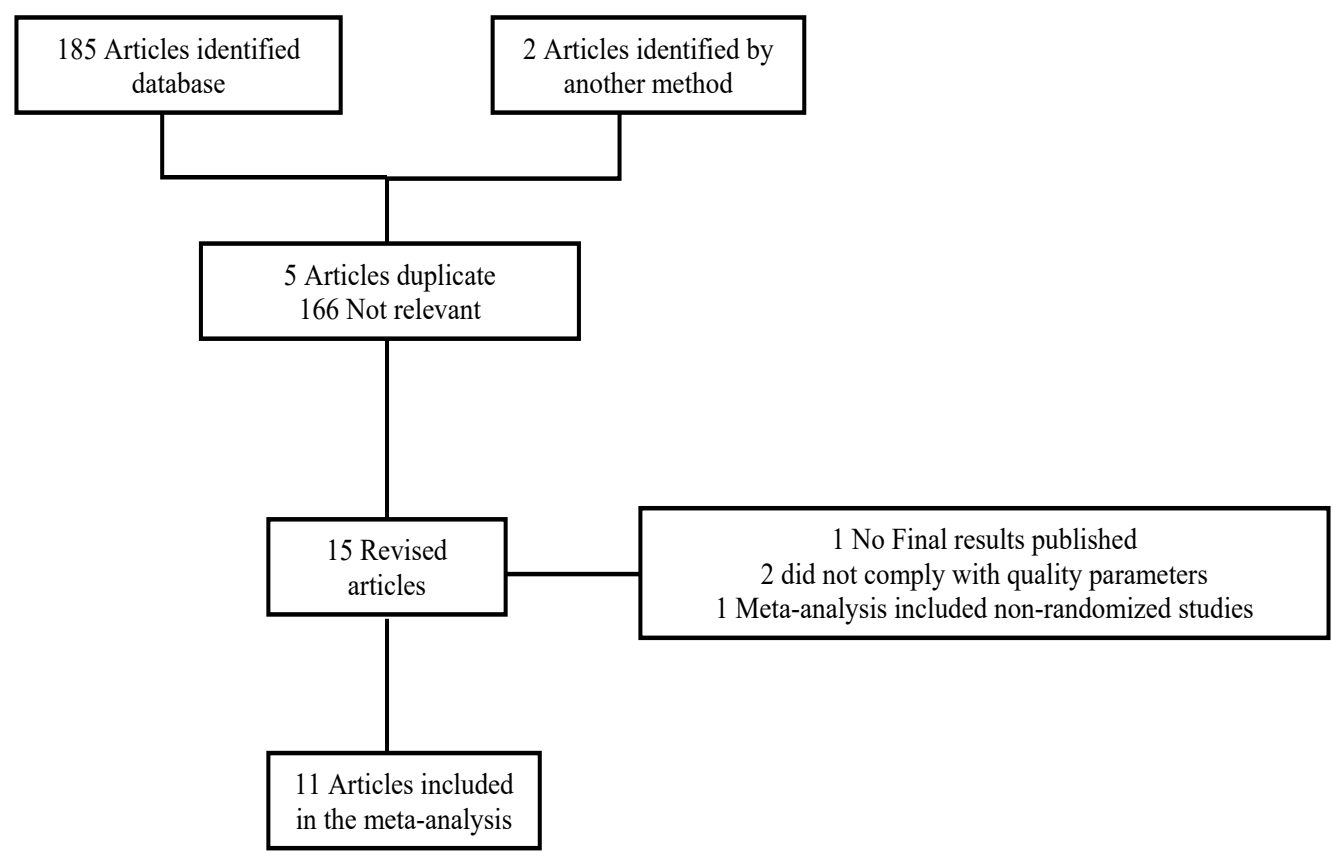

Figure 1: PRISMA Diagram 


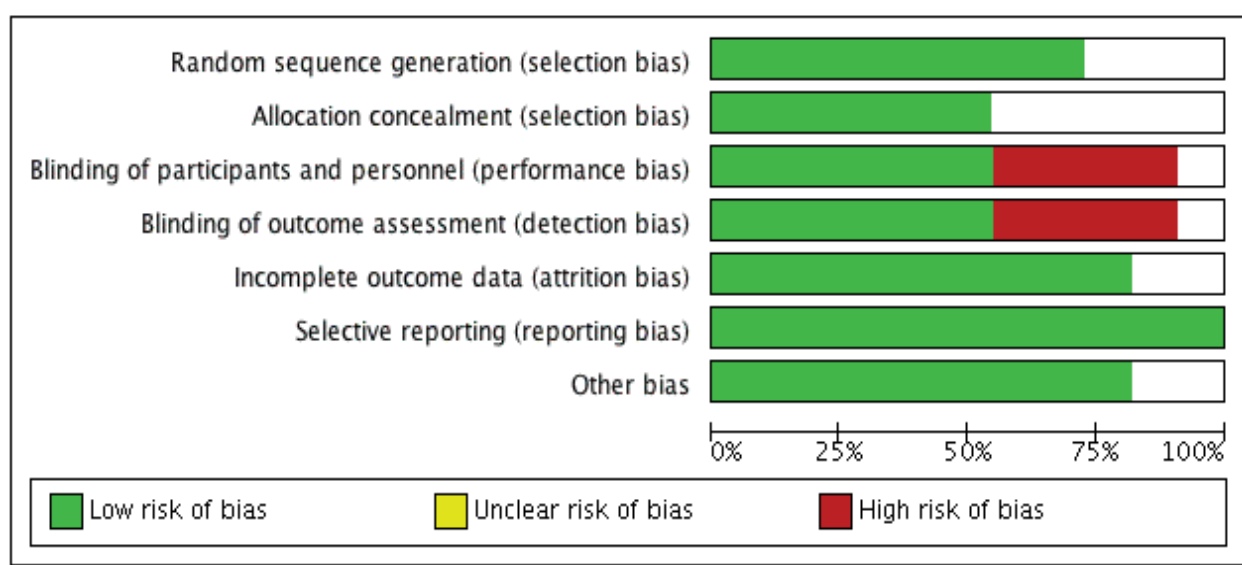

Figure 2: Risk of bias

Table 1: Characteristics of studies [11-20,28]

\begin{tabular}{|c|c|c|c|c|c|c|c|c|}
\hline Studies & $\begin{array}{r}P a \\
\text { Levo } \\
C\end{array}$ & $\begin{array}{l}\text { is } \\
\text { Idan } \\
\text { l }\end{array}$ & $\begin{array}{c}\text { Bolo } \\
\mu g / k g\end{array}$ & $\begin{array}{l}\text { Infusion } \\
\mu \mathrm{g} / \mathrm{kg} / \mathrm{m}\end{array}$ & $\begin{array}{c}\text { Duration } \\
\text { Hours }\end{array}$ & Interval & Control & Follow up \\
\hline Altenberger 2014 & 63 & 57 & - & 0.2 & 6 & Bi-week & Placebo & 24 Weeks \\
\hline Berger 2007 & 39 & 36 & 12 & 0.1 & 24 & Monthly & PGE-1* & 12 Months \\
\hline Mavrogeni 2007 & 25 & 25 & 6 & $0.1-0.2$ & 24 & Monthly & Placebo & 6 Months \\
\hline Silvetti 2014 & 185 & 141 & $3-12$ & $0.1-0.4$ & $6-24$ & Variable & $\begin{array}{l}\text { Placebo Dobutamine } \\
\text { PGE } 1^{*}\end{array}$ & 6-12 Months \\
\hline Malfatto 2012 & 22 & 11 & - & $0.1-0.4$ & 24 & Monthly & Furosemida & 16 Months \\
\hline Parissis 2006 & 17 & 8 & 6 & $0.1-0.4$ & 24 & 3 weeks & Placebo & 114 Days \\
\hline Yi 2015 & 245 & 208 & $6-12$ & $0.1-0.4$ & $6-24$ & Variable & $\begin{array}{l}\text { Placebo-Dobutamine } \\
\text { PGE1*, Furosemide }\end{array}$ & $\begin{array}{c}12 \text { weeks-1 } \\
\text { year }\end{array}$ \\
\hline Silvetti 2016 & 257 & 181 & $6-12$ & $0.1-0.4$ & $6-24$ & Variable & $\begin{array}{c}\text { Placebo } \\
\text { Dobutamina } \\
\text { PGE1* } \\
\text { Furosemida }\end{array}$ & $\begin{array}{l}12 \text { weeks-1 } \\
\text { year }\end{array}$ \\
\hline Bonios 2012 & 21 & 21 & - & 0,3 & 6 & Week & $\begin{array}{c}\text { Dobutamine } \\
\text { Levosimendan }\end{array}$ & 11-12 Months \\
\hline Kleber 2009 & 18 & 10 & 12 & 0.2 & 23 & Bi-week & Placebo & 12 Weeks \\
\hline Comin-Colet 2018 & 48 & 21 & - & 0.2 & 6 & Bi-week & Placebo & 12 Weeks \\
\hline
\end{tabular}

*(PGE1)-Prostaglandin E1

repeated every two or four weeks. The patients had an ejection fraction of $35 \%$ or less and III or IV New York Heart Association heart failure classification before randomization [14-28].

There was a significant reduction in mortality in patients with intermittent levosimendan infusion ( $13 \%$ vs $22 \%$ OR 0.45 CI 95\% 0.26 ; 0.78 . $p<0.003)$. The reduction in mortality persisted in the sensitivity analysis (Figure 3 ). Three studies reported rehospitalization but only one of them published results thereby only one of them was included $[16,27,28]$. Silvetti S, et al. [29] published in 2017, a meta-analysis evaluating rehospitalization rate after intermittent levosimendan infusions. In this meta-analysis a significant reduction in the number of rehospitalizations was found at 3 months ( $16 \%$ vs $35 \%$, RR 0.40 , $95 \%$ CI $0.27-0.59, P<0.001)$ but the effect was lost when a loading dose was administered. $10 \%$ vs $17 \%$, RR 0.43 , 95\% CI 0.16-1.19, $\mathrm{P}=0.10$ ). The major limitation of this meta-analysis was the heterogeneity of the information without clear definitions for the reason for hospitalization; therefore we did not include this meta-analysis in our study.

Natriuretic peptides levels were reduced in patients with intermittent levosimendan infusion. In LevoRep study [14], the largest randomized study done until the analysis date, which included advanced heart failure patients and intermittent levosimendan infusion in ambulatory patients found a $30 \%$ reduction in NT-ProBNP levels in the treatment group [14]. In the Malfatto G, et al. [18] study there was a reduction in BNP levels in the intervention group after the first infusion, persisting until the fourth week; in the Parissis JT, et al. [21] study there was a reduction in NT-proBNP levels as well. The LION-HEART study 


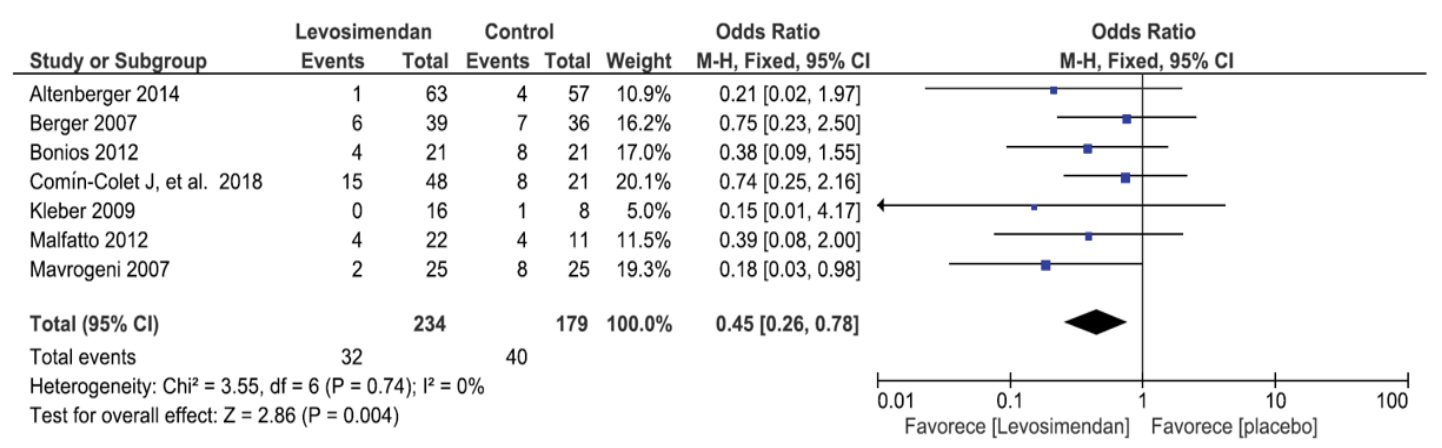

Figure 3: Outcome: Mortality

published this year demonstrated a reduction in hospitalization secondary to HF and in NT-proBNP levels with clinical stability and without significant adverse events [28]. On the other hand, in the Berger R, et al. [15] study there was no difference in the BNP or PGE1 levels at 12 weeks when analyzed independently but as a group there was a difference.

Five of the analyzed studies reported ejection fraction before and after the infusion $[15,16,18,19,21]$ and all of them demonstrated an increase between four and ten percent after the levosimendan infusion. Four of the studies reported significant differences but it was not possible to analyze this data due to the heterogeneity of the information.

\section{Discussion}

Heart failure is associated with an increase in mobility and mortality, frequent hospitalizations and a reduced quality of life, particularly in patients who are not candidates for heart transplantation or LVAD. Previous meta-analysis suggested a reduction in mortality with intermittent infusions of levosimendan but they have limitations because they included non-randomized trials or unpublished studies. Our meta-analysis excluded these studies looking for the best analysis of the levosimendan effect on mortality. This analysis demonstrated a significant reduction in mortality with intermittent levosimendan infusion, natriuretic peptide levels and increase in ejection fraction.

The limitations of our meta-analysis are the relative small number of studies and patients analyzed and the heterogeneity in the infusion protocols with variable comparators (dobutamine, furosemide, placebo). Only seven studies reported mortality outcomes. Albeit these limitations, the results are consistent with previous meta-analysis supporting the use of levosimendan in patients with advanced heart failure for a reduction in mortality.

Until the date this meta-analysis was done there was not enough data to show a reduction in hospitalization with levosimendan infusions, although descriptive studies suggest some benefit, and this could be evaluated in future investigations. There was a publication by Silvetti S, et al. [29] evaluating hospitalizations but this study was not included in our meta-analysis due to the heterogeneity of the population as well as the heterogeneity of the comparators. There was not enough information to evaluate improvement in quality of life questionnaires or costs.

The major limitation in the use of levosimendan is that is an expensive drug. With our results, together with previous publications, demonstrating a reduction in mortality we suggest that levosimendan should be considered when treating patients with advanced heart failure that are excluded for therapies as LVAD o cardiac transplantation.

\section{Conclusions}

The use of intermittent levosimendan infusions in patients with advanced heart failure is associated with a significant reduction in mortality, natriuretic peptide levels and ejection fraction. There was not enough data to evaluate other outcomes in this study.

\section{References}

1. Yancy CW, Jessup M, Bozkurt B, Butler J, Casey DE Jr, et al. (2013) 2013 ACCF/AHA guideline for the management of heart failure: a report of the American College of Cardiology Foundation/American Heart Association Task Force on Practice Guidelines. J Am Coll Cardiol 62: e147-e239.

2. Yi GY, Li JX, Zhang J, Niu LL, Zhang CY (2015) Repetitive infusion of levosimendan in patients with chronic heart failure: a meta-analysis. Med Sci Monit 21: 895-901.

3. Papp Z, Édes I, Fruhald S, De Hert SG, Salmenperä M, et al. (2012) Levosimendan: molecular mechanisms and clinical implications: consensus of experts on the mechanisms of action of levosimendan. Int J Cardiol 159: 82-87.

4. González-Chon O, López SMDCG, Mercado MAC, Sanchez EAA, Zapata REV (2005) Levosimendan: A new strategy in the treatment of heart failure. Arch Cardiol Méx 75: 130-139.

5. Kivikko M, Antila S, Eha J, Lehtonen L, Pentikainen PJ (2002) Pharmacokinetics of levosimendan and its metabolites during and after a 24-hour continuous infusion in patients with severe heart failure. Int J Clin Pharmacol Ther 40: 465-471.

6. Follath F, Cleland JG, Just H, Papp JG, Scholz H, et al. (2002) Efficacy and safety of intravenous levosimendan compared with dobutamine in severe low-output heart failure (the LIDO study): a randomised double-blind trial. Lancet 360: 196-202.

7. Moiseyev VS, Põder P, Andrejevs N, Ruda MY, Golikov AP, et al. (2002) Safety and efficacy of a novel calcium sensitizer, levosimendan, in patients with left ventricular failure due to an acute myocardial infarction. A randomized, placebo-controlled, double-blind study (RUSSLAN). Eur Heart J 23: 1422-1432.

8. Mebazaa A, Nieminen MS, Packer M, Cohen-Solal A, Kleber FX, et al. (2007) Levosimendan vs dobutamine for patients with acute decompensated heart failure: the SURVIVE Randomized Trial. JAMA 297: 1883-1891. 
9. McMurray JJ, Adamopoulos S, Anker SD, Auricchio A, Böhm M, et al. (2012) ESC guidelines for the diagnosis and treatment of acute and chronic heart failure 2012: The Task Force for the Diagnosis and Treatment of Acute and Chronic Heart Failure 2012 of the European Society of Cardiology. Developed in collaboration with the Heart Failure Association (HFA) of the ESC. Eur J Heart Fail 14: 803-869.

10. Gong B, Li Z, Yat Wong PC (2015) Levosimendan Treatment for Heart Failure: A Systematic Review and Meta-Analysis. J Cardiothorac Vasc Anesth 29: 1415-1425.

11. Liberati A, Altman DG, Tetzlaff J, Mulrow C, Gøtzscheet PC, et al. (2009) The PRISMA statement for reporting systematic reviews and meta-analyses of studies that evaluate healthcare interventions: explanation and elaboration. BMJ 339: b2700.

12. Scottish Intercollegiate Guidelines Network (2014) SIGN 50: a guideline developer's handbook. Edinburgh, Scotland.

13. Higgins JPT, Green S (2011) Cochrane Handbook for Systematic Reviews of Interventions. John Wiley \& Sons.

14. Altenberger J, Parissis JT, Costard-Jaeckle A, Winter A, Ebner C, et al. (2014) Efficacy and safety of the pulsed infusions of levosimendan in outpatients with advanced heart failure (levoRep) study: a multicentre randomized trial. Eur J Heart Fail 16: 898-906.

15. Berger R, Moertl D, Huelsmann M, Bojic A, Ahmadi R, et al. (2007) Levosimendan and prostaglandin E1 for uptitration of beta-blockade in patients with refractory, advanced chronic heart failure. Eur J Heart Fail 9: 202-208.

16. Bonios MJ, Terrovitis JV, Drakos SG, Katsaros F, Pantsios C, et al. (2012) Comparison of three different regimens of intermittent inotrope infusions for end stage heart failure. Int J Cardiol 159: 225 229.

17. Kleber FX, Bollmann T, Borst MM, Costard-Jäckle A, Ewert R, et al. (2009) Repetitive dosing of intravenous levosimendan improves pulmonary hemodynamics in patients with pulmonary hypertension: results of a pilot study. J Clin Pharmacol 49: 109-115.

18. Malfatto G, Della Rosa F, Villani A, Rella V, Branzi G, et al. (2012) Intermittent levosimendan infusions in advanced heart failure: favourable effects on left ventricular function, neurohormonal balance, and one-year survival. J Cardiovasc Pharmacol 60: 450-455.

19. Mavrogeni S, Giamouzis G, Papadopoulou E, Thomopoulou S, Dritsas A, et al. (2007) A 6-month follow-up of intermittent levosimendan administration effect on systolic function, specific activity questionnaire, and arrhythmia in advanced heart failure. J Card Fail 13: 556-559.
20. Nieminen MS, Altenberger J, Ben-Gal T, Böhmer A, Comin-Colet $\mathrm{J}$, et al. (2014) Repetitive use of levosimendan for treatment of chronic advanced heart failure: clinical evidence, practical considerations, and perspectives: an expert panel consensus. Int J Cardiol 174: 360-367.

21. Parissis JT, Adamopoulos S, Farmakis D, Filippatos G, Paraskevaidis I, et al. (2006) Effects of serial levosimendan infusions on left ventricular performance and plasma biomarkers of myocardial injury and neurohormonal and immune activation in patients with advanced heart failure. Heart 92: 1768- 1772.

22. Silvetti S, Greco T, Di Prima AL, Mucchetti $M$, de Lurdes CM, et al. (2014) Intermittent levosimendan improves mid-term survival in chronic heart failure patients: meta-analysis of randomised trials. Clin Res Cardiol 103: 505-513.

23. Silvetti S, Nieminen MS (2016) Repeated or intermittent levosimendan treatment in advanced heart failure: An updated meta-analysis. Int J Cardiol 202: 138-143.

24. Gui-yan Yi, Jun-xia Li, Zhang J, Li-li Niu, Zhang CY (2015) Repetitive Infusion of Levosimendan in Patients with Chronic Heart Failure: A Meta-Analysis. Med Sci Monit 21: 895-901.

25. Nanas JN, Papazoglou P, Tsagalou EP, Ntalianis A, Tsolakis E, et al. (2005) Efficacy and safety of intermittent, long-term, concomitant dobutamine and levosimendan infusions in severe heart failure refractory to dobutamine alone. Am J Cardiol 95: 768-771.

26. Papadopoulou EF, Mavrogeni SI, Dritsas A, Cokkinos DV (2009) Assessment of quality of life using three activity questionnaires in heart failure patients after monthly, intermittent administration of levosimendan during a six-month period. Hellenic J Cardiol 50: 269-274.

27. Levin R, Porcile R, Tanus E, Degrange M (2009) Abstract 3815: the intermittent infusion of levosimendan reduces mortality and readmissions in patients with advanced heart failure. Circ 120: S865.

28. Comín-Colet J, Manito N, Segovia-Cubero J, Delgado J, García Pinilla JM, et al. (2018) Efficacy and safety of intermittent intravenous outpatient administration of levosimendan in patients with advanced heart failure: the LION-HEART multicentre randomised trial. Eur J Heart Fail.

29. Silvetti S, Belletti A, Fontana A, Pollesello P (2017) Rehospitalization after intermittent levosimendan treatment in advanced heart failure patients: a meta-analysis of randomized trials. ESC Heart Fail 4: 595-604. 